\title{
838 THE ROLE OF MICROBIOTA IN METASTATIC BRAIN TUMORS
}

Sarah Johnson, Golnaz Morad*, Nadim Ajami, Jennifer Wargo, Matthew Wong, Matthew Lastrapes. UT MD Anderson Cancer Center, Houston, USA

Background Despite the substantial advances in the treatment of systemic cancer, brain metastases are still responsible for significant morbidity and mortality, necessitating a better understanding of the mechanisms underlying this disease. Microbiota has emerged as a significant hallmark of cancer. Our group and others have demonstrated a prominent role for gut and intratumoral microbiota in tumorigenesis, tumor immunity, and response to treatment. However, the role of microbiota in brain metastasis is poorly understood. We hypothesize that distinct microbial communities can alter the immune microenvironment in the brain and modulate the different steps of brain metastasis formation.

Methods To explore the role of microbiota in brain metastasis, we evaluated the gut and oral microbial signatures in brain metastasis patients through shotgun metagenomics sequencing. Furthermore, we conducted mechanistic in vivo studies in which the gut microbiota was depleted in conventionally raised mice using a broad-spectrum non-absorbable antibiotic regimen. Subsequently, melanoma tumor cells were injected intracranially to evaluate the effect of gut microbiota depletion and associated immune changes on tumor growth. Tumor growth was measured through in vivo bioluminescent imaging and histology. Peripheral and tumor immune profiling was conducted through flow cytometry and immunohistochemistry. Results Our clinical studies demonstrated the enrichment of distinct bacterial and viral taxa within the gut and oral microbiota in brain metastasis patients. Depletion of the gut microbiota in mice decreased tumor growth in the brain. Evaluation of the peripheral and tumor immune profiles suggested the underlying mechanisms to involve alterations in the circulating cytokine profiles and an increase in anti-tumor $\mathrm{T}$ cell activity.

Conclusions Our clinical studies suggest the association of distinct microbial communities with brain metastasis. Our preclinical findings demonstrate that the absence of gut microbiota can modulate the regulation of $\mathrm{T}$ cell activity to induce an anti-tumor response in the brain. Further studies, currently in progress, will determine the mechanistic role of dysbiotic microbiota and distinct microbial communities in this process.

Acknowledgements This work was supported by the National Institute of Health (1F32CA260769-01).

http://dx.doi.org/10.1136/jitc-2021-SITC2021.838 